\title{
Mamíferos del departamento del Chocó, depositados en las tres colecciones científicas más importantes de los Estados Unidos*
}

\author{
Mammals of the department of Choco, deposited at the three \\ main collections of the United States
}

\author{
Hugo Mantilla-Meluk ${ }^{1}$, Alex Mauricio Jiménez-Ortega ${ }^{2}$ \\ Resumen
}

\begin{abstract}
Objetivo: Complementar la información disponible sobre la diversidad de mamíferos del departamento del Chocó. Métodos: Se analizaron registros museológicos de especímenes chocoanos depositados en las tres colecciones más relevantes de los Estados Unidos: American Museum of Natural History de Nueva York (AMNH, 39 spp/127 registros), Field Museum of Natural History de Chicago (FMNH, 53 spp/374 registros) y el United State National Museum of the Smithsonian Institution de Washington DC (USNM, $19 \mathrm{spp} / 65$ registros). Para tener un punto de evaluación de la representatividad del material analizado fuera de Colombia, los registros recuperados fueron contrastados con aquellos registros chocoanos depositados en la colección de mamíferos del Instituto de Ciencias Naturales de la Universidad Nacional de Colombia (ICN, 52 spp/721 registros). Resultados: Producto de este análisis se generó una base de datos consistente en 566 registros, para un total de 82 especies de mamíferos chocoanos depositados en colecciones extranjeras. Estos registros necesitan una revisión urgente que clarifique su estatus taxonómico y de esta manera los ponga al servicio de la comunidad científica y los tomadores de decisiones a nivel local. Según los registros recuperados, se identifica como llamativa la presencia del primate endémico de Colombia Saguinus oedipus, para el departamento del Chocó, soportada por material depositado en el AMNH. De igual manera se reportan registros del murciélago embalonúrido, Cormura brevirostris y el perezoso Choloepus didactylus, que de ser verificados en su taxonomía representarían extensiones significativas en la distribución conocida de estos taxones y sus primeros registros en colecciones confirmados para el departamento. Llama también la atención por su afinidad biogeográfica registros del murciélago nectarívoro Hylonycteris underwoodi y el cuzumbo Nasua narica candace, conocidos de Centro América, cuya distribución es posible que se extienda en el territorio chocoano. Finalmente, aparece como interesante el registro de una mandíbula identificada como perteneciente a un manatí (Trichechus manatus) proveniente del municipio de Unguía y depositada en el FMNH, como la única muestra almacenada en colecciones de referencia.
\end{abstract}

Palabras clave: Bases de datos, Colecciones científicas, Mamíferos, Registros museológicos.

\begin{abstract}
Objective: To complete the available information on biodiversity of the department of Choco. Methods: We analyzed museological records of Chocoan specimens deposited in the three main collections of the United States: American Museum of Natural History de Nueva York (AMNH, 39 spp/127 records); Field Museum of Natural History de Chicago (FMNH, 53 spp/374 records); y el United State National Museum of the Smithsonian Institution de Washington DC (USNM, $19 \mathrm{spp} / 65$ records). To evaluate the importance of of the analyzed material we contrasted the recovered records with Chocoan records deposited at the collection of the Instituto de Ciencias Naturales of the Universidad Nacional de Colombia (ICN, 52 spp/721 registros). Results: As product of our analyses we generated a database of 566 records representing a total of 82 Chocoan mammalian species deposited in scientific collections of the United States. The analyzed records require an urgent revision of their taxonomic status, in order to make them a useful source of
\end{abstract}

* Proyecto «Desarrollo de herramientas de gestión para el posicionamiento de la biodiversidad como fuente de bienestar social y ambiental en el Chocó, occidente colombiano».

Programa de Biología, Universidad del Quindío, Armenia, Quindío, Colombia. e-mail: hugo.mantillameluk@gmail.com

2 Grupo de Investigación Manejo de Fauna Silvestre Chocoana, Facultad de Ciencias Naturales, Programa de Biología, Universidad Tecnológica del Chocó, Quibdó, Chocó, Colombia. e-mail: alexmauriciojimenez@gmail.com

Fecha recepción: Noviembre 29, $2014 \quad$ Fecha aprobación: Marzo 21, 2015 Editor asociado: Palacios L

(c) Rev. Biodivers. Neotrop. 2015; 5 (2): 124-30 
information for local decision makers. We highlight the presence of the Colombian endemic primate Saguinus oedipus, for department of Choco, supported by material deposited at the $\mathrm{AMNH}$. In the same manner we report the presence of the emballonurid bat Cormura brevirostris, and the slot Choloepus didactylus, that if taxonomically confirmed will represent significant distribution extensions and first confirmed records in collections of these taxa for the department. We also highlight the records of the nectarivorous bat Hylonycteris underwoodi and the coati Nasua narica candace, both species known from Central America and potentially present in the department. Finally, called our attention the presence of a mandible of the manatee (Trichechus manatus) from the municipio of Unguia deposited at the FMNH; this represents the only museum voucher material of the species from this portion of the Colombian territory.

Keywords: Databases, Mammals, Museological records, Scientific collections.

\section{Introducción}

Con casi medio millar de especies, Colombia ocupa la cuarta posición mundial y la segunda en el neotrópico en términos de biodiversidad de mamíferos (Alberico et al. 2000; Solari et al. 2013), destacándose en particular por su riqueza los quirópteros para los cuales Colombia ostenta el primer lugar en número de especies en la región neotropical superando a países de mayor dimensión como Brasil, México y Perú (Mantilla-Meluk et al. 2009). Tanto la diversidad geográfica como ecológica que caracteriza a Colombia son parte de un escenario en el que se han dado procesos evolutivos únicos que en conjunto han brindado múltiples oportunidades para la generación de nuevas especies y el establecimiento de complejos arreglos de las mismas a lo largo de la extensión del país. Es así como el territorio colombiano se presenta como un privilegiado y único laboratorio natural para el estudio de los procesos evolutivos y ecológicos que explican la diversidad y los patrones de distribución de este grupo de vertebrados (Mantilla-Meluk et al. 2009). Para Colombia, en particular el levantamiento de los Andes y la compleción del Istmo de Panamá, han resultado en la generación de un andén de aislamiento físico y climático sobre la porción pacífica del territorio nacional, en el denominado Chocó Biogeográfico, área que mantiene niveles de pluviosidad entre los más altos del planeta, soportando así ecosistemas caracterizados por bosques estratificados hiperhúmedos. Para Colombia, el andén Pacífico in- cluye los departamentos de Cauca, Valle del Cauca, Chocó y Antioquia, siendo el departamento del Chocó el que mayor área cubre de esta región.

A pesar de su especialidad ecosistémica, el estudio continuo de la diversidad de mamíferos del departamento del Chocó corresponde a esfuerzos relativamente recientes, que señalan a esta porción de Colombia como una de las regiones más diversas para este grupo de vertebrados. Los altos niveles de diversidad biológica contenidos en esta región imponen altísimas responsabilidades de carácter global para la sociedad colombiana y en particular para los tomadores de decisiones locales en el Chocó, en términos de la generación de los procesos y las dinámicas que propendan por el entendimiento y conservación de este importante elemento del patrimonio diverso de la nación. En este sentido, identificamos las evaluaciones regionales y locales detalladas, como una herramienta ineludible en la identificación de los tensores que afectan diferencialmente la diversidad. Como se mencionó, una fracción muy significativa de la diversidad de mamíferos de Colombia se encuentra contenida en la región pacífica en el Chocó Biogeográfico. De las 492 especies reconocidas en la lista más reciente y comprensiva de mamíferos de Colombia, 195 (39.6\%) están contenidas en la región pacífica, con un total de 109 especies cuya ocurrencia ha sido verificada para el territorio del departamento del Chocó (Solari et al. 2013). Sorprende el bajo porcentaje de registros de mamíferos depositados en colecciones colombianas, para el departamento del Chocó, cuando su territorio corresponde a una de las áreas más diversas de la geografía del país, como lo demuestran los análisis de esfuerzo de muestreo de mamíferos en Colombia (Mantilla-Meluk y Jiménez-Ortega 2006, Mantilla-Meluk et al. 2009).

La documentación de la mastofauna de Colombia ha incluido el esfuerzo no solo de las instituciones colombianas, sino que ha tenido la participación de importantes instituciones extranjeras que organizaron expediciones extensivas e intensivas en áreas de especial interés biogeográfico del país, sobre todo en la primera mitad del siglo XX (Mantilla-Meluk et al. 2014).

Dentro del conjunto de instituciones científicas que han desarrollado trabajo de documentación en Colombia se destacan los tres principales museos de historia natural de los Estados Unidos: Ameri- 
can Museum of Natural History, de Nueva York (AMNH), Field Museum of Natural History, de Chicago (FMNH) y National Museum of Natural History del Smithsonian Institution en Washington (USNM). Expediciones en la región pacífica del territorio de Colombia fueron conducidas por equipos especializados de estas tres instituciones, resultando en material museológico de incalculable valor científico que infortunadamente ha sido poco estudiado por investigadores nacionales.

En su propósito de realizar una documentación detallada de la diversidad mastozoológica contenida en el territorio chocoano, la Universidad Tecnológica del Chocó ha identificado este vacío y ha definido como uno de los ejes la revisión y sistematización de la información sobre mamíferos chocoanos depositados en colecciones científicas del país y el exterior, con énfasis en colecciones asociadas con instituciones en los Estados Unidos. Este trabajo busca generar una herramienta comprensiva de manejo de la información sobre la diversidad mastozoológica chocoana, representada por material depositado en colecciones extranjeras con el fin de identificar tanto los vacíos de información en cubrimiento geográfico, así como errores de distribución y errores taxonómicos de determinación para este set de datos, de manera que se identifiquen prioridades en investigación taxonómica a ser desarrolladas de manera presencial, en una segunda fase que incluya la visita física a las colecciones mencionadas y la revisión sistemática de este material así como establecer mecanismos de repatriación de material y/o información asociada con los mismos.

\section{Materiales y métodos}

Un total de 566 registros de mamíferos chocoanos correspondientes a material museológico depositado en las tres colecciones científicas más relevantes de los Estados Unidos: American Museum of Natural History de Nueva York (AMNH), Field Museum of Natural History de Chicago (FMNH) y el United State National Museum of the Smithsonian Institution de Washington DC (USNM), fueron compilados y evaluados con el fin de complementar la lista comprensiva de mamíferos de Colombia producida por Solari et al. (2013). El desarrollo de esta herramienta y su análisis permitirá una optimización de los tiempos de revisión, derivada de la priorización de temas de investigación, a la vez que será material de soporte a la repatriación digital del material museológico del departamento del Chocó depositado en colecciones fuera del territorio nacional. Con el fin de establecer la relevancia de la información analizada en colecciones extranjeras se hace una comparación preliminar de registros con aquellos depositados en la colección del Instituto de Ciencias Naturales de la Universidad Nacional de Colombia (ICN) en Bogotá.

\section{Resultados y discusión}

Representatividad de los registros extranjeros analizados. Según este análisis, un total de 82 especies de mamíferos chocoanos, se encuentran depositadas en las colecciones analizadas así: AMNH (39 spp/127 registros), USNM (19 spp/65 registros), FMNH (53 spp/374 registros); 63 de estas especies, hacen parte de las 109 cuya ocurrencia ha sido verificada para el territorio del departamento del Chocó según Solari et al. (2013) (Tabla 1). Mientras la colección de mamíferos del ICN cuenta a la fecha con un total de 721 registros chocoanos, los registros extranjeros depositados en las tres colecciones mayores de los Estados Unidos sumaron 566 registros, es decir $78 \%$ en número con respecto a esta colección.

Llama la atención la colección de mamíferos chocoanos depositados en el Field Museum de Chicago (FMNH), la cual supera en número de especies (FMNH 53 spp/374 registros) a la colección colombiana del Instituto de Ciencias Naturales (ICN, 52 $\mathrm{spp} / 721$ registros), considerada la de mayor representatividad de la biodiversidad del país (Tabla 1).

Identificación de prioridades en análisis sistemáticos y taxonómicos. Para algunas de las especies con material depositado en las colecciones analizadas (Tabla 1), se requieren análisis más detallados que corroboren su identificación taxonómica, porque representan novedades que potencialmente pueden corresponder a extensiones en distribución como el murciélago insectívoro Cormura brevirostris y el perezoso Choloepus didactylus, que de ser verificados en su taxonomía representarían extensiones significativas en la distribución conocida de estos taxones y sus primeros registros confirmados para el departamento. De manera análoga, se reporta la presencia del murciélago nectarívoro Hylonycteris underwoo- 
Tabla 1. Lista de las especies documentadas para el territorio del Chocó en las colecciones del American Museum of Natural History; Field Museum of Natural History (FMNH); el National Museum of Natural History (USNM); y el Instituto de Ciencias Naturales de la Universidad Nacional (ICN)

\begin{tabular}{|c|c|c|c|c|}
\hline & FMNH & AMNH & USNM & ICN \\
\hline $\begin{array}{l}\text { Número de especies } \\
\text { Didelphimorphia }\end{array}$ & 53 & 39 & 19 & 52 \\
\hline Caluromys derbianus derbianus & $x$ & & & \\
\hline Chironectes minimus minimus & $x$ & $x$ & & \\
\hline Didelphis marsupialis ${ }^{\circ}$ & $x$ & $x$ & & \\
\hline Gracilinanus agilis & $x$ & & & \\
\hline Marmosa isthmica & $x$ & & & \\
\hline Marmosa phaea & $x$ & & & \\
\hline Philander opossum melanurus & $x$ & $x$ & & $x$ \\
\hline Metachirus nudicaudatus colombianus & $x$ & & & \\
\hline Total & 8 & 3 & 0 & 1 \\
\hline \multicolumn{5}{|l|}{ Pilosa } \\
\hline Bradypus variegatus & $x$ & & & \\
\hline Bradypus variegatus infuscatus & & $x$ & & \\
\hline Choloepus didactylus ** & $x$ & & & \\
\hline Choloepus hoffmanni capitalis & & $\mathrm{x}$ & & $\mathrm{x}$ \\
\hline Cyclopes didactylus & $x$ & & & \\
\hline Tamandua mexicana & $\mathrm{x}$ & & & \\
\hline Tamandua tetradactyla chiriquiensis & & $x$ & & \\
\hline Total & 4 & 3 & 0 & 1 \\
\hline \multicolumn{5}{|l|}{ Sirenia } \\
\hline Trichechus manatus ${ }^{* *}$ & $x$ & & & \\
\hline $\begin{array}{r}\text { Total } \\
\end{array}$ & 1 & 0 & 0 & 0 \\
\hline \multicolumn{5}{|l|}{ Chiroptera } \\
\hline Artibeus lituratus palmarum & & $x$ & $\mathrm{x}$ & \\
\hline Artibeus jamaicensis & & & $x$ & $x$ \\
\hline Artibeus planirostris & & & & $x$ \\
\hline Carollia brevicauda & & $x$ & $x$ & $x$ \\
\hline Carollia castanea & & $x$ & $x$ & $x$ \\
\hline Carollia perspicillata & $x$ & $x$ & $x$ & $x$ \\
\hline Chiroderma trinitatum ${ }^{\circ \circ}$ & & & & $\mathrm{x}$ \\
\hline Chiroderma villosum ${ }^{\circ}$ & & & & $x$ \\
\hline Choeroniscus periosus periosus & & $x$ & & \\
\hline Cormura brevirostris & $x$ & & & \\
\hline Cynomops abrasus & $x$ & & & \\
\hline Dermanura glauca* & & $x$ & $x$ & $\mathrm{x}$ \\
\hline Dermanura phaeotis* & & & $x$ & $x$ \\
\hline Desmodus rotundus ${ }^{\circ 0}$ & & & & $x$ \\
\hline Diaemus youngi ${ }^{\circ \circ}$ & & & & $\mathrm{x}$ \\
\hline Enchisthenes hartii ${ }^{\circ}$ & & & & $\mathrm{x}$ \\
\hline Furipterus horrens & & $x$ & & \\
\hline Glossophaga soricina & & & $x$ & $x$ \\
\hline Glossophaga commissarisi ${ }^{\circ}$ & & & & $\mathrm{x}$ \\
\hline Hylonycteris underwoodi underwoodi & $x$ & & & \\
\hline Lichonycteris obscura & $x$ & & & $x$ \\
\hline Lonchophylla mordax ${ }^{\circ \circ}$ & & & & $\mathrm{x}$ \\
\hline Lonchophylla robusta & & $x$ & & $x$ \\
\hline Lophostoma silvicolum $^{\circ}$ & & & & $\mathrm{x}$ \\
\hline Messophylla macconnelli & & & $x$ & \\
\hline Micronycteris megalotis megalotis & $x$ & & & \\
\hline Myotis nigricans ${ }^{\circ}$ & $x$ & $\mathrm{x}$ & & $\mathrm{x}$ \\
\hline Molossus molossus & $x$ & & $\mathrm{x}$ & $x$ \\
\hline
\end{tabular}


Tabla 1. Lista de las especies documentadas para el territorio del Chocó en las colecciones del American Museum of Natural History; Field Museum of Natural History (FMNH); el National Museum of Natural History (USNM); y el Instituto de Ciencias Naturales de la Universidad Nacional (ICN) (continuación)

\begin{tabular}{|c|c|c|c|c|}
\hline & FMNH & AMNH & USNM & ICN \\
\hline Molossus pretiosus & & & $x$ & \\
\hline Noctilio albiventris minor & $x$ & $x$ & & $x$ \\
\hline Noctilio leporinus ${ }^{\circ 0}$ & & & & $x$ \\
\hline Phyllostomus discolor discolor & $\mathrm{x}$ & $\mathrm{x}$ & & \\
\hline Phyllostomus hastatus ${ }^{\circ}$ & $x$ & $\mathrm{x}$ & $x$ & $x$ \\
\hline Platyrrhinus chocoensis & & $\mathrm{x}$ & & \\
\hline Platyrrhinus helleri helleri & & $\mathrm{x}$ & $x$ & $x$ \\
\hline Rhinophylla alethina & & $\mathrm{x}$ & & \\
\hline Rhynchonycteris naso & $x$ & & & $x$ \\
\hline Saccopteryx bilineata & $x$ & & & $x$ \\
\hline Saccopteryx leptura ${ }^{\circ}$ & & & & $\mathrm{x}$ \\
\hline Thyroptera sp. ${ }^{\circ}$ & & & & $x$ \\
\hline Uroderma bilobatum convexum & $x$ & $x$ & & $x$ \\
\hline Uroderma magnirostrum ${ }^{\circ}$ & & & & $x$ \\
\hline Vampyressa nymphaea & & $x$ & $x$ & $x$ \\
\hline Vampyressa pusilla ${ }^{\circ 0}$ & & & & $x$ \\
\hline Vampyrodes caraccioli ${ }^{\circ \circ}$ & & & & $x$ \\
\hline Total & 14 & 17 & 14 & 33 \\
\hline \multicolumn{5}{|l|}{ Carnivora } \\
\hline Bassaricyon medius medius & $\mathrm{x}$ & & & \\
\hline Felis concolor ${ }^{\circ \circ}$ & & & & $x$ \\
\hline Felis silvestris $^{\circ \circ}$ & & & & $x$ \\
\hline Eira barbara sinuensis & $x$ & & & $\mathrm{x}$ \\
\hline Lontra longicaudis annectens & $x$ & & & \\
\hline Nasua nasua candace ${ }^{* *}$ & $x$ & & & \\
\hline Mustela frenata affinis & & & $x$ & \\
\hline Potos flavus megalotus & $x$ & $x$ & & \\
\hline Procyon cancrivorus panamensis & $x$ & & & \\
\hline Total & 6 & 1 & 1 & 3 \\
\hline \multicolumn{5}{|l|}{ Artiodactyla } \\
\hline Mazama temama* & $x$ & & & $x$ \\
\hline Pecari tajacu & $x$ & & & $\mathrm{x}$ \\
\hline Total & 2 & 0 & 0 & 2 \\
\hline \multicolumn{5}{|l|}{ Cetaceae } \\
\hline Stenella attenuata ${ }^{\circ \circ}$ & & & & $x$ \\
\hline Tursiops truncatus ${ }^{\circ \circ}$ & & & & $x$ \\
\hline Total & 0 & 0 & 0 & 2 \\
\hline \multicolumn{5}{|l|}{ Primates } \\
\hline Alouatta palliata aequatorialis & $x$ & & & \\
\hline Alouatta seniculus seniculus & $x$ & & & \\
\hline Aotus zonalis* & $x$ & & & $x$ \\
\hline Aotus trivirgatus ${ }^{\circ}$ & & & & $x$ \\
\hline Ateles geoffroyi ${ }^{\circ}$ & & & & $x$ \\
\hline Cebus capucinus capucinus & $x$ & $\mathrm{x}$ & & \\
\hline Saguinus geoffroy & $x$ & & & $x$ \\
\hline Saguinus oedipus ** & & $\mathrm{x}$ & & \\
\hline Total & 5 & 2 & 0 & 4 \\
\hline \multicolumn{5}{|l|}{ Rodentia } \\
\hline Heteromys australis australis & & $\mathrm{x}$ & & \\
\hline Heteromys desmarestianus & $\mathrm{x}$ & & & \\
\hline Hoplomys gymnurus & $x$ & $x$ & & \\
\hline Cuniculus paca guanta & $x$ & $\mathrm{x}$ & $x$ & $x$ \\
\hline
\end{tabular}


Tabla 1. Lista de las especies documentadas para el territorio del Chocó en las colecciones del American Museum of Natural History; Field Museum of Natural History (FMNH); el National Museum of Natural History (USNM); y el Instituto de Ciencias Naturales de la Universidad Nacional (ICN) (continuación)

\begin{tabular}{|c|c|c|c|c|}
\hline & FMNH & AMNH & USNM & ICN \\
\hline Dasyprocta punctata colombiana & $x$ & $x$ & $x$ & $x$ \\
\hline Melanomys caliginosus & & $x$ & & $x$ \\
\hline \multicolumn{5}{|l|}{ Mesoplodon densirostris } \\
\hline Microsciurus alfari fusculus & & & $x$ & \\
\hline Microsciurus mimulus & $x$ & & & \\
\hline Microsciurus flaviventer & & $x$ & & \\
\hline Microsciurus isthmius** & & $x$ & & \\
\hline Neacomys tenuipes tenuipes & $x$ & $x$ & & \\
\hline Oligoryzomys fulvescens & $\mathrm{x}$ & & & \\
\hline Transandinomys talamancae* & $x$ & $x$ & & \\
\hline Proechimys semispinosus & $x$ & $x$ & & $\mathrm{x}$ \\
\hline Rattus rattus rattus ${ }^{\star *}$ & & $x$ & & \\
\hline Rhipidomys sp. ${ }^{\circ \circ}$ & & & & $x$ \\
\hline Sciurus morulus* & $\mathrm{x}$ & $x$ & $\mathrm{x}$ & \\
\hline Sciurus granatensis ${ }^{* *}$ & & $x$ & & \\
\hline Sigmodontomys alfari & $\mathrm{x}$ & & & $\mathrm{x}$ \\
\hline Zygodontomys brevicauda cherriei & $x$ & & & \\
\hline Total & 12 & 13 & 4 & 6 \\
\hline \multicolumn{5}{|l|}{ Lagomorpha } \\
\hline Sylvilagus brasiliensis nicefori & $x$ & & & \\
\hline Total & 1 & 0 & 0 & 0 \\
\hline
\end{tabular}

di y el cuzumbo Nasua narica candace, especies conocidas de Centro América, que potencialmente extenderían con estos registros su distribución en el territorio chocoano. Asimismo se requiere revisar el estatus taxómico de los ejemplares de la zarigueya Didelphis marsupialis y el murciélago insectívoro Myotis nigricans, para los cuales se reporta más de una subespecie para el departamento del Chocó en las colecciones analizadas.

Aunque Millan (1999) menciona la presencia del manatí (Trichechus manatus) y Savage et al. (2003) del mono titi (Saguinus oedipus) para la zona norte del Chocó, las muestras depositadas en el FMNH y el AMNH para cada especie respectivamente, representan las únicas muestras provenientes del Chocó, existentes en colecciones científicas de referencia.

Diez y nueve especímenes chocoanas depositadas en las colecciones analizadas se encontraron solamente determinados a género, incluyendo quirópteros: Molossus, Platyrrhinus, Thyroptera, y Uroderma; los géneros de roedores Neacomys, Proechimys, Rhipidomys, y artiodáctilo Tayassu.

Un total de 64 de los registros analizados repre- sentan una incongruencia entre especie y distribución, no siendo recocidas las especies en las que fueron clasificados como presentes en el Chocó (Solari et al. 2013). Estas incongruencias pueden estar asociadas con identificaciones erróneas (lo más probable para el registro del murciélago frugívoro Carollia subrufa), también con cambios en la nomenclatura (lo más probable para registros del murciélago frugívoro Dermanura cinerea y el venado Mazama americana).

Adicionalmente, a este material depositado en las colecciones extranjeras, se señala como importante el registro del ratón de cola de pincel género Rhipidomys, con registros chocoanos en la colección del ICN. Roedores del género Rhipidomys cuentan con muy pocos registros para esta región.

\section{Conclusiones}

A pesar de que varias instituciones han adelantado esfuerzos muy importantes en documentar la mastofauna chocoana, como la Universidad Tecnológica del Chocó, la Universidad del Valle y la Universidad de 
Antioquia, este estudio se centra el análisis comparativo con la colección del ICN, al ser la colección con mejor representatividad regional a nivel país. En ningún momento se quiere desconocer el valor incalculable del material chocoano depositado en las instituciones antes mencionadas. Es por ello que este trabajo se identifica como una aproximación preliminar, que hace parte de una investigación más detallada que se adelanta en este instante, en la que se da un cubrimiento museológico.

Este análisis permite evidenciar varios aspectos importantes que llaman la atención sobre la documentación de mamíferos del territorio nacional. Existe un número significativo de registros de mamíferos en colecciones fuera del país. Estos registros corresponden en su mayoría a expediciones anteriores a 1960, que tuvieron un cubrimiento importante de la geografía del país, abarcando localidades que no han sido cubiertas por los esfuerzos nacionales. Esto es importante porque muchos de estos registros pueden representar extensiones de distribución de las especies, e incluso nuevos registros para Colombia. Las afinidades taxonómicas de muchos de estos especímenes aún no han sido plenamente determinadas y requieren de un examen comparativo detallado. Afortunadamente, la mayor parte de este material esta preservado en buenas condiciones y tiene datos asociados que multiplican su valor científico. Es importante recordar que mucho de este material fue recolectado por colectores profesionales pagados por instituciones de amplia tradición museológica.

De acuerdo con todo lo anterior, listados regionales que no han incluido revisiones de los especímenes extranjeros pueden estar sesgando su aproximación a la descripción de la diversidad de mamíferos. Se sugiere como importante el empezar a delinear una política de repatriación física o virtual de especímenes depositados en colecciones extranjeras que permitan el esclarecimiento de las afinidades taxonómicas de las especies de Colombia y la mejor determinación de sus patrones de distribución. La documentación sistemática de los mamíferos de Colombia que incluye la validación de especies y la descripción de nuevas formas se ha dificultado en el país debido a la ausencia de programas de financiamiento que permita visitar las colecciones extranjeras que no solo contienen un número importante de muestras del país, sino que permite la comparación con ejemplares provenientes de otros países. Se reconoce el valor de iniciativas como Global Biodiversity Information Facility (GBIF) y el Sistema de Información sobre Biodiversidad (SIB) que facilitan la minera de datos.

\section{Agradecimientos}

Este trabajo se realizó gracias al apoyo y financiamiento del proyecto "Desarrollo de herramientas de gestión para el posicionamiento de la biodiversidad como fuente de bienestar social y ambiental en el Chocó, occidente colombiano". Los autores agradecen a los editores y revisores por sus aportes. Agradecemos a BD Paterson del FMNH, K Helgen y D Wilson del USNM y H López del ICN, por la asistencia en el trabajo museológico.

\section{Literatura citada}

Alberico M, Cadena A, Hernández-Camacho JI, Muñoz-Saba Y. 2000. Mamíferos (Synapsida:Theria) de Colombia. Biota Colomb 1: 43-75.

Mantilla-Meluk H, Ramírez-Chave HE, Jiménez-Ortega AM, Rodríguez-Posada ME. 2014. Emballonurid bats from Colombia, annotated checklist, distribution and biogeography. Therya. 5 (1): 627-53.

Mantilla-Meluk H, Jiménez-Ortega AM, Baker RJ. 2009. Phyllostomid bats of Colombia: Annotated checklist, distribution, and biogeography. Special Publications. Museum of Texas Tech University 56: 1-44.

Mantilla-Meluk H, Jiménez-Ortega AM. 2006. Estado de conservación y algunas consideraciones biogeográficas sobre la quiropterofauna del Chocó Biogeográfico colombiano. Revista Institucional, Universidad Tecnológica del Chocó Diego Luis Córdoba. 25: 10-7.

Millán SL. 1999. Estado de salud del manatí (Trichechus sp) en Colombia. Tesis MSc, Universidad de Puerto Rico, Mayagüez; $129 \mathrm{pp}$.

OMACHA y CODECHOCÓ. 2008. Diagnóstico y acciones de conservación del manatí Trichechus mantas y la babilla Caiman crocodilus en la cuenca media y baja del Atrato, Chocó-Colombia. Quibdó: OMACHA y CODECHOCÓ; $136 \mathrm{p}$.

Savage A, Giraldo H, Soto LH, García FE, Nassar-Montoya F. 2003. Proyecto Titi: Establecimiento de técnicas de campo para el monitoreo a largo plazo del iiti cabeza blanca (Saguinus oedipus) en Colombia. En: Pereira-Bengoa Pp. 40-70, Nassar-Montoya F, \& Savage A (Eds.). Primatología del Nuevo Mundo. Bogotá: Centro de Primatología Araguatos Ltdá. pp. 40-70.

Solari S, Muñoz-Saba Y, Rodríguez-Mahecha JV, Defler TR, Ramírez-Chaves HE, Trujillo F. 2013. Riqueza endemismo y conservación de los mamíferos de Colombia. Mastozool Neotrop. 20 (2): 301-65. 